\title{
Clinicopathological Features and Outcome for Dogs with Mast Cell Tumors and Bone Marrow Involvement
}

\author{
L. Marconato, G. Bettini, C. Giacoboni, G. Romanelli, A. Cesari, A. Zatelli, and E. Zini \\ Background: Mast cell tumors (MCTs) with bone marrow (BM) involvement are poorly documented in dogs and are as- \\ sociated with a poor prognosis. Successful treatment strategies have not been described. \\ ficial. \\ Hypothesis: Clinicopathologic findings of affected dogs are not specific. Administration of lomustine or imatinib is bene- \\ Animals: Fourteen dogs with MCT and BM involvement. \\ Methods: Clinical and laboratory evaluations were performed in each dog on admission and during follow-up. All dogs \\ received prednisone. Additionally, 8 dogs received lomustine and 3 dogs received imatinib. Imatinib was administered if tumor- \\ associated tyrosine kinase KIT was aberrant. \\ Results: On admission, 11 dogs had a single cutaneous nodule and 3 dogs had multiple nodules. Involvement of regional \\ lymph nodes, liver, or spleen was observed in each dog. BM infiltration with mast cells (MCs) was observed in all dogs. On \\ CBC, nonregenerative anemia, leukopenia, or thrombocytopenia was common. Four dogs had circulating MCs. Increased \\ alkaline phosphatase or alanine transferase activity was observed in 12 and 10 dogs, respectively. Treatment with lomustine \\ induced partial remission in 1 of 8 dogs. Median survival time was 43 days (range, 14-57). Dogs on imatinib experienced com- \\ plete remission. Two dogs survived for 117 and 159 days, and the third was alive after 75 days. Dogs treated symptomatically \\ did not improve and were euthanized after 1, 14, and 32 days. \\ Conclusions and Clinical Importance: A combination of clinical and laboratory evaluation helps in identifying dogs with \\ MCT and BM infiltration. Administration of lomustine is not helpful in affected dogs. The beneficial effect of imatinib warrants \\ further investigation.
}

Key words: Canine; Mastocytoma; Molecular-targeted therapy; Systemic metastasis.

$\mathbf{M}$ ast cell tumors (MCTs) have extremely variable biologic behavior in dogs, ranging from strictly benign cutaneous masses to metastatic disease. ${ }^{1-3}$ Some dogs with cutaneous MCTs have no clinical signs, but those with visceral and bone marrow (BM) involvement may suffer from serious disease. ${ }^{4,5}$ Clinical signs are mainly related to the release of mast cell (MC) mediators, including histamine, heparin, and other vasoactive amines, which can lead to localized flushing, gastrointestinal signs, generalized pruritus, vasodilatory shock, and anaphylaxis. ${ }^{6}$ Moreover, MC infiltration itself may cause organomegaly, impairment of organ function, and, in the case of BM involvement, pancytopenia. ${ }^{4}$

MCs are rarely observed in BM cytology samples obtained from healthy dogs, ${ }^{4,7}$ and they may be increased in dogs with diseases other than MCTs, such as those with inflammatory skin diseases, enteritis, pancreatitis, heartworm disease, and parvovirus infection. ${ }^{8}$ In MCT, the

From the Clinica Veterinaria L'Arca, Naples, Italy (Marconato, Giacoboni); the Department of Veterinary Public Health and Animal Pathology, University of Bologna, Ozzano Emilia, Italy (Bettini, Cesari); the Clinica Veterinaria Nerviano, Milano, Italy (Romanelli); the Clinica Veterinaria Pirani, Reggio Emilia, Italy (Zatelli); and the Clinic for Small Animal Internal Medicine, Vetsuisse Faculty, University of Zürich, Zürich, Switzerland (Zini).

Findings of this study were presented in part at the Veterinary Cancer Society-European Society of Veterinary Oncology Joint Meeting, Copenhagen, Denmark, 2008.

Corresponding author: Laura Marconato, Clinica Veterinaria L'Arca, Vico Cacciottoli 46/47, 80129 Naples, Italy; e-mail: lauramarconato@yahoo.it.

Submitted November 13, 2007; Revised January 16, 2008; Accepted April 23, 2008.

Copyright (C) 2008 by the American College of Veterinary Internal Medicine

$10.1111 / j .1939-1676.2008 .0128 . x$ presence of neoplastic BM infiltration is rare, and it is generally more common in dogs with aggressive (grade III) primary cutaneous tumors. ${ }^{3,4,-11}$ In a recent retrospective study, the incidence of BM infiltration at initial staging in dogs with cutaneous MCTs was $2.8 \%{ }^{12}$ An additional retrospective study ${ }^{13}$ focused on the incidence of BM disorders in dogs. Among BM samples, metastatic MCs were found in $0.4 \%$ of specimens. ${ }^{13}$

Pertinent literature has emerged in the last decade with regard to therapeutic strategies for canine MCTs, covering several approaches such as surgery, radiation therapy, chemotherapy, electrochemotherapy, and photodynamic therapy.,14-17 Among chemotherapeutic drugs, lomustine and vinblastine are considered the most active agents against neoplastic MCs. ${ }^{18-22}$ In recent years, the use of targeted drugs in the treatment of MCT has also been proposed. ${ }^{23,24}$

MCs are derived from hematopoietic precursor cells that reside in BM and peripheral blood. The process of $\mathrm{MC}$ differentiation is influenced by cytokines and a stem cell factor (SCF) that represents the ligand for KIT, a transmembrane tyrosine kinase (TK) receptor encoded by the proto-oncogene $c$-kit and constitutively expressed by MCs. ${ }^{25}$ In recent years, $c$-kit has been implicated in the pathogenesis of canine MCTs. ${ }^{25}$ In $15-50 \%$ of dogs with MCT, c-kit mutations, especially in exon 11, have been detected and are considered responsible for ligand-independent KIT phosphorylation and for the subsequent autonomous proliferation of MCs. ${ }^{24-30} \mathrm{Ad}-$ ditionally, a significant association of $c$-kit mutations with higher histologic grade and worse prognosis has been shown in dogs with MCTs. ${ }^{25,28,30}$ Recent studies have also suggested that KIT autophosphorylation leads to aberrant cytoplasmic localization of KIT, compared with MCTs that lack mutations and have normal peri- 
membrane protein localization. ${ }^{30,31}$ Furthermore, aberrant KIT protein localization has been found to be significantly associated with increased cellular proliferation. ${ }^{30}$

Recently, a number of potent TK inhibitors have been developed, with imatinib mesylate being among the most explored in humans. ${ }^{32}$ Imatinib directly inhibits the catalytic activity of KIT by interfering with the binding of adenosine triphosphate, thereby blocking TK phosphorylation and downstream signaling. ${ }^{32}$ Until now, few studies have investigated the efficacy of imatinib for treatment of MCTs in veterinary medicine, and most studies have been performed in cats or with canine cell culture lines. ${ }^{31,33-35}$ The optimal drug dosage and duration of treatment await investigation in dogs. Conversely, another TK inhibitor (SU11654) has been shown to have antiproliferative effects in canine MCT biopsy samples and measurable effects in dogs with different tumors, including MCTs. ${ }^{23,24}$

A review of the literature failed to identify studies on treatments specifically aimed to eradicate MCTs with $\mathrm{BM}$ infiltration. Currently, disease progression in dogs with $\mathrm{BM}$ involvement is rapid and is associated with fatal outcome in most cases. ${ }^{4}$ Given the fact that cytopenias are common in affected dogs, the use of chemotherapy becomes more difficult and needs to be carefully balanced against adverse effects on BM cell progenitors.

Consequently, the primary aims of this study were to describe the clinicopathological features of canine MCT with $\mathrm{BM}$ infiltration and the clinical outcome of affected dogs treated with prednisone, lomustine, or imatinib. Furthermore, the antitumoral activity of the above-mentioned therapeutic agents and the development of toxicity were assessed in treated dogs.

\section{Materials and Methods \\ Enrollment Criteria}

Dogs with cutaneous MCTs, either solitary or multiple, and cytologically proven BM infiltration (stage IV based on the World Health Organization system for canine MCTs $)^{36}$ were eligible for recruitment. Dogs with second malignancies as well as those with concurrent nonneoplastic disorders, possibly contributing to systemic reactive mastocytosis, ${ }^{8}$ were excluded. In addition, dogs that received either chemotherapy or radiation therapy in the preceding 6 months were excluded. Finally, dogs were excluded from the study if they did not undergo complete staging.

Initial staging included history and physical examination, CBC with differential, serum biochemistry, liver function tests (pre- and postprandial bile acids), coagulation profile (activated partial thromboplastin time [aPTT], prothrombin time [PT], and plasma fibrinogen concentration), urinalysis (including urine protein to creatinine ratio), cytological evaluation of the cutaneous nodule(s) and regional lymph node(s), 3-dimensional measurement of the cutaneous nodule(s), thoracic radiographs (3 views), abdominal ultrasound examination, fine-needle aspirates of liver and spleen, and cytologic examination of BM obtained from the iliac crest.

Regarding visceral involvement, liver and spleen were considered to have metastatic disease if cytologic samples showed sheets or clusters of MCs. Isolated MCs were considered neoplastic if atypical on morphology or in the case of aberrant (cytoplasmic) KIT (CD117) expression.

No published information indicates how to determine whether the BM is affected by metastatic disease, and a clear cut-off number for BM MCs has not been established. According to a previous study, ${ }^{4} \mathrm{BM}$ was considered infiltrated if $>10 \mathrm{MCs}$ were present per 1,000 nucleated cells. In a more recent study, BM was considered infiltrated if $>3-5 \%$ of all nucleated cells (ANC) were identified as MCs on cytology smears. ${ }^{21}$ Therefore, for the diagnosis of BM involvement to be made with confidence, we arbitrarily decided that MCs had to represent $>10 \%$ of ANC in cytologic smears or, if atypical, $>5 \%$ of ANC. Slides from each BM aspirate were stained with May Grünwald Giemsa (MGG) and examined for the presence of MCs. The number of MCs was expressed as a percentage of the total count of ANC.

In selected cases (specifically in those dogs whose owners considered targeted treatment), an antibody against the KIT receptor (CD117) was applied to cytologic samples of BM to evaluate the pattern of KIT expression in neoplastic MCs. Briefly, air-dried BM smears were postfixed in acetone and incubated overnight with rabbit anti-human CD117 polyclonal antibody ${ }^{\mathrm{a}}$ (1:100); a streptavidinbiotin labeling system ${ }^{\mathrm{a}}$ was used for immunolabeling, and the reaction was visualized with diaminobenzidine. A modified MGG stain (May-Grünwald solution for 1 minute, Giemsa solution diluted $1 \mathrm{drop} / \mathrm{cm}^{3}$ for 5 minutes) was used as a counterstain instead of hematoxylin to better differentiate MCs from immature myeloid cells that also can express CD117.

\section{Treatment Designs}

At the time of investigation, treatment with imatinib was very expensive; therefore, dogs were not randomly assigned to treatment groups. Rather, treatment group assignment was made on the basis of owner cost concern as follows: single-agent prednisone ${ }^{\mathrm{b}}$; lomustine and prednisone; or imatinib and prednisone. Regardless of the chosen treatment, all dogs received supportive care with the PO administration of $\mathrm{H}_{1}$ (chlorpheniramine) ${ }^{\mathrm{c}}$ and $\mathrm{H}_{2}$ (ranitidine) ${ }^{\mathrm{d}}$ histamine-blocking drugs. Prednisone was administered at a dosage of $20 \mathrm{mg} / \mathrm{m}^{2}$ PO q12h; this dosage was gradually tapered and discontinued over 24 weeks. Drugs were started after staging was completed. All owners gave written informed consent.

Dogs receiving prednisone were re-evaluated at monthly intervals by means of CBC, serum biochemistry, BM examination, and abdominal ultrasound examination until death or euthanasia.

Lomustine $^{\mathrm{e}}$ was administered at a dosage of $60 \mathrm{mg} / \mathrm{m}^{2}$ PO q28d. Because of possible myelosuppression and cumulative hepatic toxicity, $\mathrm{CBC}$ as well as liver enzyme activity and liver function tests were repeated 14 days after treatment and immediately before administration of the next dose of the drug. The other laboratory tests as well as complete staging were repeated at monthly intervals. The lomustine dosage was maintained for the duration of the study unless toxicity occurred or disease progressed.

Imatinib $^{\mathrm{f}}$ was administered at the dosage of $4.4 \mathrm{mg} / \mathrm{kg}$ PO q24h at the 1st morning meal. In cats, imatinib mesylate has been administered at dosages ranging from 5 to $10 \mathrm{mg} / \mathrm{kg},{ }^{34}$ but similar information is not available for dogs. According to preclinical toxicology studies in dogs, imatinib administered PO at dosages $\geq 10 \mathrm{mg} / \mathrm{kg}$ was associated with gastrointestinal, hepatic, and hematologic toxicity, whereas a dosage of $3 \mathrm{mg} / \mathrm{kg}$ appeared to be safe. ${ }^{37}$ Considering the above, we decided to treat dogs at a dosage of $4.4 \mathrm{mg} / \mathrm{kg}$. Because tumoral expression of the $c-k i t$ gene product was required for this group of animals, KIT expression (including both intensity and localization) was established at baseline evaluation for each dog treated with imatinib. Dogs were treated for at least 1 month before evaluation of efficacy and safety. If treatment with imatinib was beneficial, treatment was continued until progression of disease or death. Complete staging was repeated at monthly intervals, whereas $\mathrm{CBC}$ and serum biochemistry were repeated biweekly. 


\section{Response Criteria}

To evaluate response, clinical, radiologic, ultrasonographic, and laboratory investigation were repeated at the following time points: monthly during the first 6 months and every 3 months thereafter. Examinations were performed more frequently if deemed necessary. Furthermore, any new masses were evaluated cytologically. Response definitions included complete remission (CR), partial remission (PR), stable disease (SD), and progressive disease (PD). $\mathrm{CR}$ was characterized by disappearance of $\mathrm{MC}$ infiltrates in the involved sites at follow-up evaluation, absence of progression of systemic mastocytosis at other sites, active hematopoiesis with $<10$ MCs per 1,000 nucleated cells on BM cytology, and full recovery of blood counts with no detectable MCs on blood smears and a white blood cell count within normal limits. PR was defined as incomplete regression of $\mathrm{MC}$ infiltrates, with reduction of $\mathrm{BM} \mathrm{MCs}$ by $\geq 50 \%$ and to $<10 \%$, CBC recovery with the persistence of few peripheral MCs $(<5 \%)$, reduction of hepatosplenomegaly, and $\geq 50 \%$ decrease in 1 dimension in the cutaneous nodules. SD was defined as $<50 \%$ decrease in 1 dimension, but no more than a $10 \%$ increase in any dimension of all measurable tumors, with marrow MCs reduced by $<50 \%$. PD was defined as $\geq 10 \%$ increase in any dimension of measurable tumor with no reduced or increased marrow MCs. Relapse was defined as cytologic evidence of MCT in any anatomical site in dogs that had CR, whereas relapse for animals with PR was defined as progression. Overall, survival was defined as the time between the 1st day of treatment and death.

\section{Toxicity Evaluation}

For Groups 2 and 3, adverse events related to treatment were assessed according to the guidelines of the Veterinary Co-Operative Oncology Group. ${ }^{38}$

In general, in dogs experiencing Grade 2 nonhematological toxicity, treatment (either imatinib or lomustine) was withheld until toxicity had resolved to Grade $\leq 1$. In dogs having recurrent toxicity, treatment was withheld as above and the next dose was decreased by $25 \%$. In dogs experiencing Grades 3-4 nonhematological toxicity, the study drug was withheld until the toxicity had resolved to Grade $\leq 1$, and the dose then was decreased by $50 \%$.

For hematological toxicity, no dose reductions or interruptions were performed for Grades 1-2 toxicity, whereas in patients experiencing Grades 3-4 toxicity, treatment was withheld until toxicity was reduced to Grade $\leq 2$, and then resumed at the same dose. If Grades 3-4 toxicity recurred, treatment was withheld.

\section{Results}

\section{Patient and Tumor Characteristics}

Between April 2004 and August 2007, 14 dogs with previously untreated MCT and BM involvement were enrolled. There were 7 crossbreeds, 2 Rottweiler, and 1 each for Chow Chow, Labrador Retriever, Pekingese, German Shepherd, and Siberian Husky. Median age was 10 years (range, 16 months- 15 years). Five dogs were intact males, 5 intact females, and 4 spayed females. Median body weight was $24.8 \mathrm{~kg}$ (range, 4.5-36.1).

Two dogs had no clinical signs (substage a), whereas the remaining 12 were symptomatic (substage b). The duration of clinical signs before referral ranged from 10 to 124 days (median, 21 days). Reported clinical signs were often vague and included lethargy in 10 dogs, decreased appetite in 9 , weight loss in 7 , vomiting in 4 , and diarrhea or polyuria and polydipsia in 2 each. The major complaint for 1 dog was melena, whereas 2 dogs showed intense generalized pruritus. The presenting clinical sign in another dog was straining to urinate and defecate.

At diagnosis, 11 dogs had a single cutaneous nodule and 3 had multiple nodules. MCTs were cytologically well differentiated or of intermediate differentiation in 4 dogs each or undifferentiated in 6 dogs. Regional lymph node involvement was cytologically confirmed in 10 dogs, and hepatic and splenic involvement was evident in 11 and 10 dogs, respectively. Three dogs had pulmonary involvement, as evidenced by a generalized peribronchial interstitial pattern in the caudodorsal lung fields in $1 \mathrm{dog}$, and by multiple pulmonary nodules in the others, and confirmed in 2 dogs by postmortem histopathologic evaluation, and in 1 dog by computed tomographic-guided cytologic examination. One dog had bilateral renal involvement.

Examination of BM identified infiltration of MCs in all dogs, ranging from scattered aggregates to complete effacing of the normal hematopoietic architecture (7$85 \%$ of ANC). Five dogs had normal CBC despite BM infiltration $(7-33 \%)$.

Regarding $\mathrm{CBC}$, at initial evaluation the median packed cell volume (PCV) was 32\% (range, 12-51; reference range, 37-55). Three dogs had severe anemia $(\mathrm{PCV}<15 \%), 2$ had moderate anemia (PCV 15-20\%), and 4 had mild anemia (PCV 21-36\%). Anemia was nonregenerative in all affected dogs (reticulocytes $<40,000 / \mu \mathrm{L})$. Initially, the median white blood cell count was $7,800 / \mu \mathrm{L}$ (range, 2,600-12,900; reference range, $6,000-17,000)$. Moderate eosinophilia was present in 3 dogs, with a median value of 2,100 eosinophils $/ \mu \mathrm{L}$ (range, 1,950-2,350; reference range, 100-1,250). In 4 dogs, circulating MCs were found on blood smear evaluation, making up $2-10 \%$ of nucleated blood cells. Thrombocytopenia was observed in 5 dogs (median, $95,000 / \mu \mathrm{L}$; range, 86,000-108,000; reference range, $120,000-350,000)$. Coagulation parameters were prolonged in 3 dogs, expressed as a $20 \%$ increase in PT and aPTT, but none of them had concurrent thrombocytopenia or increased fibrinogen concentration, excluding disseminated intravascular coagulation. Alkaline phosphatase activity (ALP) was increased in 12 dogs (median, $460 \mathrm{U} / \mathrm{L}$; range, 202-790; reference, 0-160), and alanine transferase (ALT) was increased in 10 dogs (median, $78 \mathrm{U} / \mathrm{L}$; range, 68-122; reference range, 0-65). Liver dysfunction was not observed.

\section{Treatment Effects and Safety Data}

At the end of the study, all dogs but one were dead with a median survival time of 41 days (range, 1-159). Response rates for all dogs are shown in Table 1 .

Single-Agent Prednisone. Three dogs received singleagent prednisone with no improvement of clinical status. All of them experienced PD, eventually leading to euthanasia after 1, 14, and 32 days, respectively.

Lomustine and Prednisone. In addition to prednisone, $8 \mathrm{dogs}$ were treated with lomustine at a dosage of $60 \mathrm{mg}$ / $\mathrm{m}^{2}$. The median number of lomustine treatments was 1 (range, 1-2). The original protocol was designed to last 6 months, but in all cases treatment was stopped earlier 
Table 1. Clinical outcome.

\begin{tabular}{rlllr}
\hline ID & \multicolumn{1}{c}{ Treatment } & Response & $\begin{array}{c}\text { TTF/DFI } \\
\text { (days) }\end{array}$ & $\begin{array}{r}\text { Survival } \\
\text { (days) }\end{array}$ \\
\hline 1 & Single-agent prednisone & PD & No response & 1 \\
2 & Single-agent prednisone & PD & No response & 14 \\
3 & Single-agent prednisone & PD & No response & 32 \\
4 & Lomustine & PD & No response & 14 \\
5 & Lomustine & PD & No response & 44 \\
6 & Lomustine & PD & No response & 47 \\
7 & Lomustine & PD & No response & 57 \\
8 & Lomustine & PD & No response & 21 \\
9 & Lomustine & PD & No response & 41 \\
10 & Lomustine & PD & No response & 30 \\
11 & Lomustine & PR & 41 & 46 \\
12 & Imatinib & CR & 78 & 117 \\
13 & Imatinib and lomustine & CR & 150 & 159 \\
14 & Imatinib and lomustine & CR & $75^{\text {a }}$ & Alive \\
\hline
\end{tabular}

${ }^{\mathrm{a}} \mathrm{Still}$ in CR.

TTF, time to treatment failure; DFI, disease-free interval; PD, progressive disease; $\mathrm{PR}$, partial remission; $\mathrm{CR}$, complete remission.

because of PD. Clinical improvement did not occur in most cases. Dog 11 achieved PR of 41 days duration after the 1st lomustine dose, but was eventually euthanized 5 days later after sudden worsening of its general condition, most likely because of massive degranulation, as assessed by hyperalgesia, edema of the neck and front legs, and massive gastrointestinal bleeding.

Toxicity induced by lomustine was evaluated at each visit. Five dogs experienced marked nonfebrile neutropenia by the 1 st examination, 14 days after chemotherapy was started (median, 800 neutrophils $/ \mu \mathrm{L}$; range, 300-1,200). Among them, only 2 had peripheral cytopenia at presentation, including anemia $(\mathrm{n}=2)$ and thrombocytopenia $(n=1)$. With the exception of Dog 4 , for which euthanasia was requested by the owner at 14 days because of poor life quality, in the others treatment had to be delayed because of neutropenia. Unfortunately, in none of them could the 2nd dose of lomustine be administered because the disease was rapidly progressive, leading to euthanasia. Three dogs experienced no toxicity and received the 2 nd dose of lomustine at the scheduled time. With regard to serum biochemistry, increase in ALP and ALT activities was present at diagnosis in 7 of 8 dogs and enzyme activity did not increase during the study period. The median survival time for all dogs was 43 days (range, 14-57 days).

Imatinib and Prednisone. Three dogs were treated PO with imatinib at a daily dosage of $4.4 \mathrm{mg} / \mathrm{kg}$, in addition to prednisone.

At presentation, the 1st dog had BM infiltration, with MCs accounting for $33 \%$ of ANC. Nonetheless, CBC indicated no abnormalities. MCs infiltrating the BM were round and highly granulated and showed marked CD117 cytoplasmic staining. The dog demonstrated rapid clinical improvement with progressive disappearance of its preputial mass and metastatic inguinal lymph node and no reported drug-related toxicity. A repeated abdominal ultrasound examination 1 month after initiation of treatment disclosed substantial decrease in the size of the liver and spleen, with no MCs observed on fine-needle aspirates in both organs. Furthermore, a BM aspirate performed at this same time indicated a decrease of MCs from 33 to $2 \%$ with a normal CBC. Based on these findings, the tumor response was defined as a CR. At 44 days, the dog was still in CR, also based on a BM evaluation. Despite the excellent response, 78 days after initial presentation, the MCT on the prepuce started to grow again. On ultrasound examination, enlarged sublumbar lymph nodes and hepatosplenomegaly were detected. Furthermore, BM cytology indicated increased MCs (35\% of ANC) and peripheral mastocytemia (5\%). Because of physical deterioration and onset of clinical signs, imatinib was stopped. Despite supportive care with antibiotics (enrofloxacin ${ }^{\mathrm{g}}$ and amoxicillin/clavulanate), ${ }^{\mathrm{h}}$ antiemetics (maropitant), ${ }^{\mathrm{i}} \mathrm{H}_{1}$ (diphenhydramine) ${ }^{\mathrm{j}}$ and $\mathrm{H}_{2}$ (ranitidine) antihistamines, and prednisone, the dog required 2 hospital admissions and 1 blood transfusion over 39 days, after which euthanasia was performed. The dog survived for 117 days.

In the 2 nd case, peripheral blood was remarkable for the presence of tricytopenia and $10 \%$ circulating MCs. A BM aspirate identified marked decreases in the erythroid, myeloid, and megakaryocytic lineages, with MCs accounting for $85 \%$ of marrow cellularity and displaying strong and diffuse immunocytochemical cytoplasmic CD117 expression (Fig 1). Imatinib was started in the dog. CR was soon achieved, as assessed by disappearance of the interscapular MCT, marked size reduction of the metastatic prescapular lymph node, and normalization of $\mathrm{CBC}$. The dramatic improvement of the clinical picture was accompanied by a marked reduction in MC numbers in the BM aspirate. After 1 month of treatment, $\mathrm{BM}$ evaluation indicated a notable reduction in the extent of MC infiltration, with MCs representing $4 \%$ of ANC. Furthermore, no MCs were observed on lymph node, splenic, and hepatic cytology. At subsequent reevaluation (Day 60), no clinicopathologic abnormalities were observed, with the exception of an increased number of BM MCs $(11 \%)$. Because of this finding, lomustine at $60 \mathrm{mg} / \mathrm{m}^{2} \mathrm{PO}$ q28d was added to imatinib aiming to achieve a possible synergistic effect. By day 90 , the dog had a normal $\mathrm{CBC}$ and abdominal ultrasound examination, $1 \%$ MCs in $\mathrm{BM}$, and no cutaneous or regional lymph node relapse. CR lasted for an additional 2 months, after which the tumor relapsed in the BM, spleen, and liver, as assessed by cytologic evaluation (day 150). Because of pruritus and development of hemorrhagic diarrhea, the dog was hospitalized and supported with blood transfusion, diphenhydramine, ranitidine, and prednisone. During the following 9 days, the disease progressed with a dramatic increase in circulating and BM MCs (23 and 92\%, respectively), and the dog eventually was euthanized on day 159 .

On admission, the 3rd dog had BM-infiltrating MCs, accounting for $7 \%$ of ANC. MCs were highly atypical and showed aberrant KIT expression. No peripheral blood abnormalities were identified. The dog received daily imatinib and once monthly lomustine. Follow-up with physical examination, $\mathrm{CBC}$, serum biochemistry, abdominal ultrasound examination, thoracic radiogra- 

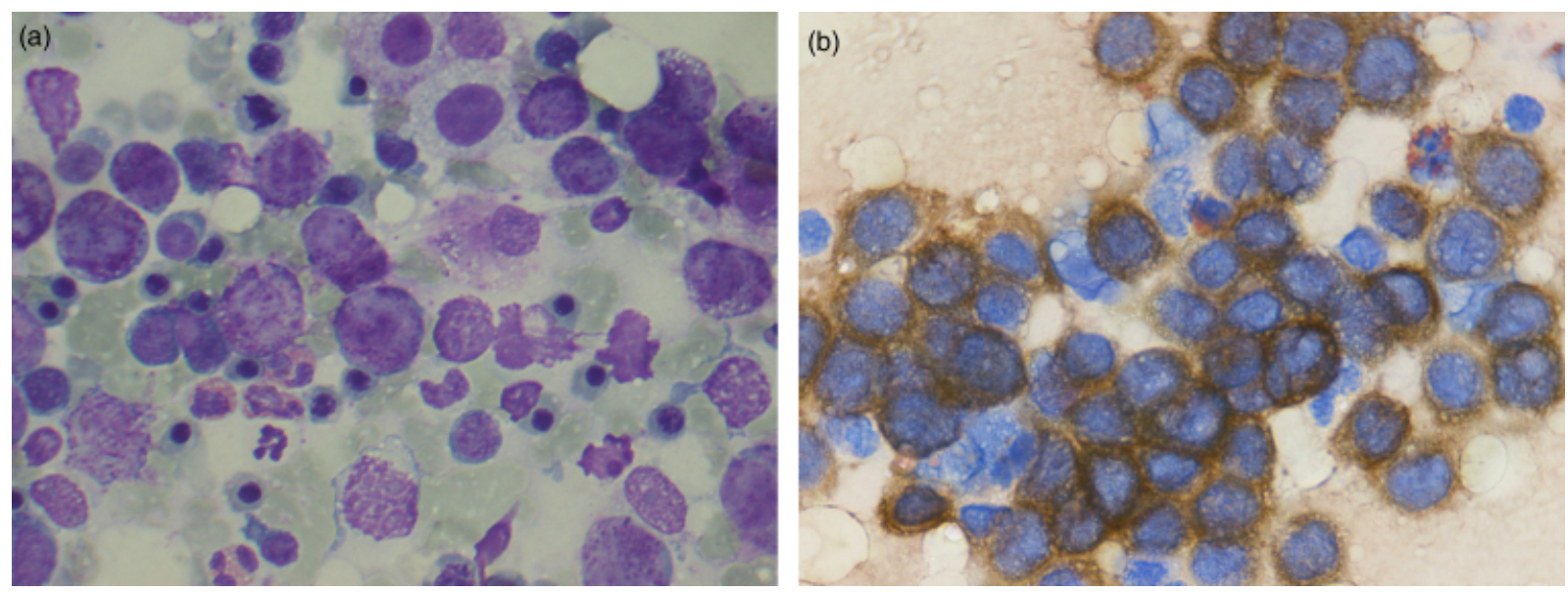

Fig 1. (a) Dog 13: bone marrow, myelophthysis. (b) Diffuse immunocytochemical cytoplasmic CD117 expression.

phy, and BM cytology indicated CR. At the time of reporting, 75 days after treatment was started, the dog was still being treated with imatinib and lomustine, showing no tumor relapse.

None of the dogs on imatinib required drug withdrawal or temporary interruption because of toxicity. Treatment was well tolerated, with no evidence of hematological or nonhematological adverse effects.

\section{Discussion}

Canine MCT with BM involvement is a rare condition for which successful treatment strategies are currently lacking, and affected dogs invariably have a poor prognosis.

In agreement with previous studies, ${ }^{4,-11}$ our findings show that MCT with BM infiltration is aggressive in dogs, with 13 of 14 animals dying because of complications of mastocytosis or drug-resistant disease, with a median survival time of 41 days. In all cases, a combination of multi organ involvement and severe BM infiltration contributed to death. The initial clinical signs were vague and mainly attributable to local or systemic effects of MC mediators and organ infiltration. Clinical manifestations indicative of BM infiltration were often difficult to recognize. Indeed, because most dogs had more than 1 organ affected, nonspecific signs such as weakness, lethargy, and weight loss made the clinical picture more complex. As a consequence, the hypothesis of $\mathrm{BM}$ involvement relied on the fact that multi organ infiltration with MCs, peripheral blood cytopenias, or both were observed in all dogs.

Dogs with MC BM infiltration have a number of hematological abnormalities, including anemia, thrombocytopenia, leukopenia, leukocytosis, eosinophilia, and basophilia. ${ }^{4,5,12}$ Circulating MCs may be observed occasionally. ${ }^{4,8,12}$ Of note is the fact that 5 dogs in the present study showed no hematological abnormalities despite marked BM involvement (with MCs representing 7$33 \%$ of ANC). This finding has important clinical implication because lack of BM examination during staging may result in an underestimation of the number of patients harboring $\mathrm{BM}$ disease, possibly leading to inadequate treatment. Unlike previous experiences in MCT staging that indicated no need for BM evaluation unless hematological abnormalities were identified, ${ }^{12}$ our results suggest that BM cytology is necessary to accurately stage MCTs, especially when considering chemotherapy. It is well known that dogs with systemic disease involving the liver, spleen, or both have poor outcome and that BM evaluation may not add prognostic information. If conventional cytotoxic chemotherapy is considered in a dog with systemic involvement, BM evaluation may be necessary. Indeed, further hematological complications may occur with myelosuppressive chemotherapy, thereby reducing life quality and possibly survival. Splenic and hepatic disease with MC infiltration is a common finding in dogs with BM mastocytosis. ${ }^{4,9-11}$ In this cohort of dogs, 13 of 14 dogs had either liver or spleen involvement. Interestingly, hepatic dysfunction was not observed, as demonstrated by normal liver function tests. The ALP and ALT activities were frequently increased at presentation. However, because the activity was stable over the study period, we believe that these increases were attributable to a combination of neoplasia-induced stress leading to endogenous glucocorticoid release and to direct neoplastic hepatic infiltration. ${ }^{39}$ The only dog with no hepatic or splenic involvement had pulmonary infiltration. Lung metastasis was also observed in 2 additional dogs, which is quite uncommon for canine MCT. Altogether, these findings emphasize the importance of thoracic radiography in the initial evaluation.

Historically, canine MCT with BM infiltration has been considered an extremely aggressive disease characterized by a rapid clinical course, with limited or no treatment options available. In recent years, much has been learned about MC biology and tumor-related genetic aberrations. ${ }^{25}$ It was shown that normal MC development and proliferation involve the action of SCF on KIT receptors. ${ }^{40}$ In some dogs with MCT, the occurrence of mutation in the $c$-kit gene leads to aberrant expression of KIT receptors, which ultimately promotes uncontrolled proliferation. ${ }^{26,27,29,30}$ Based on this finding, the identification of aberrant KIT expression may become a target for treatment of canine MCT. One drug developed to selec- 
tively inhibit TK has been assessed in dogs with different tumors. ${ }^{23,24}$ The TK inhibitor SU11654 showed antitumor activity, especially in dogs with MCT. ${ }^{24}$ However, the issue of how to treat marrow infiltration was not addressed.

Lomustine is an active agent for the treatment of canine MCT. ${ }^{3,22}$ In the study reported here, not only did lomustine not show any activity on MCs infiltrating BM, as assessed by rapid disease progression in all treated dogs, but also its use was associated with marked hematological toxicity (ie, neutropenia). Conversely, although used only in $3 \mathrm{dogs}$, imatinib was effective against MC BM infiltration, whereas neither single-agent prednisone nor lomustine improved survival. In response to imatinib therapy, the clinical signs resolved and the BM-infiltrating MCs disappeared in all affected dogs. Although single-agent prednisone may induce responses in up to $70 \%$ of dogs with MCTs, ${ }^{41}$ it is likely that imatinib partially contributed to the CR in treated dogs, because dogs receiving single-agent prednisone did not experience any objective response. Although the present results may support the use of imatinib for MCT with $\mathrm{BM}$ infiltration, acquired resistance to the drug may develop. In fact, in 2 dogs, treatment failure was observed after 78 and 150 days, respectively. During relapse, BM aspirates showed similar numbers of MCs as did specimens collected at initial presentation. In human medicine, resistance to imatinib is a major concern and is believed to be caused by mutations in the kinase domain interfering with imatinib binding. ${ }^{42}$ In addition, imatinib is a substrate of P-glycoprotein, a drug efflux pump that is highly expressed in hematopoietic stem cells, leading to low intracellular concentrations of the drug. ${ }^{42}$ To address the growing problem of relapse, molecular-targeted drugs may be integrated into preexisting therapeutic regimens, with the goal of overcoming resistance and enhancing antitumor activity through a synergistic or additive effect. In human medicine, drug combination strategies are often applied based on the evidence that combining specific inhibition of tumor signal pathways together with more standard treatment can effectively enhance anticancer activity ${ }^{43}$ In dogs, no information is available on the interaction of novel anticancer drugs with other treatment modalities, and it is possible that successful long-term treatment of MCT with $\mathrm{BM}$ infiltration may require a combination of kinase inhibitors and conventional chemotherapy. Although preliminary, the results obtained in 2 imatinib-treated dogs may support such strategy. In fact, the 1 dog that was started on imatinib and later had lomustine added experienced the longest survival, whereas the other dog that simultaneously received imatinib and lomustine did not show any evidence of a systemic or BM relapse after more than 2 months of observation. Thus, a combination of imatinib, lomustine, and prednisone may yield maximal benefit. This hypothesis needs to be tested in additional clinical trials.

With regard to imatinib toxicity, in all treated dogs the drug was well tolerated without detectable adverse effects. Furthermore, imatinib seemed to spare normal hemopoietic cells, as demonstrated by effective hemopoiesis and repopulation of metastatic BM.
Although suggestive, this investigation has some limitations, such as the small number of animals studied and the fact that dogs were not equally distributed among treatments. As suggested by the literature, and confirmed by our study, which spanned 3 years, BM infiltration is rare in dogs with MCT, which limits the number of dogs available for investigation. Also, 8 dogs received lomustine, whereas only 3 dogs received imatinib. Unfortunately, imatinib is very expensive and prohibitive for many dog owners. Future larger studies assessing the efficacy of single-agent imatinib, with or without adjuvant chemotherapy, are warranted.

\section{Footnotes}

\author{
a DakoCytomation, Glostrup, Denmark \\ ${ }^{\mathrm{b}}$ Deltacortene, Bruno-Farmaceutici, Rome, Italy \\ ${ }^{\mathrm{c}}$ Rimeton, Shering-Plough, Milan, Italy \\ ${ }^{\mathrm{d}}$ Zantac, GlaxoSmithKline, Verona, Italy \\ ${ }^{\text {e }}$ Prava, Bristol-Myers Squibb, Baar, Switzerland \\ ${ }^{\mathrm{f}}$ Glivec, Novartis Europharm Limited, Horsham West Sussex, UK \\ ${ }^{\mathrm{g}}$ Baytril, Bayer HealthCare, Milan, Italy \\ ${ }^{\text {h }}$ Synulox, Pfizer, Rome, Italy \\ ${ }^{\mathrm{i}}$ Cerenia, Pfizer \\ ${ }^{\mathrm{j}}$ Benadryl, Johnson \& Johnson, New Brunswick, NJ
}

\section{References}

1. Bostock DE. The prognosis following surgical removal of mastocytomas in dogs. J Small Anim Pract 1973;14:27-41.

2. Patnaik AK, Ehler WJ, MacEwen EG. Canine cutaneous mast cell tumour: Morphologic grading and survival time in 83 dogs. Vet Pathol 1984;21:469-474.

3. Thamm DH, Vail DM. Mast cell tumors. In: Withrow SJ, Vail DM, eds. Withrow \& MacEwen's. Small Animal Clinical Oncology, 4th ed. Philadelphia, PA: WB Saunders Co; 2007:402-424.

4. O'Keefe DA, Couto CG, Burke-Schwartz C, Jacobs RM. Systemic mastocytosis in 16 dogs. J Vet Intern Med 1987;1:75-80.

5. Takahashi T, Kadosawa T, Nagase M, et al. Visceral mast cell tumors in dogs: 10 cases (1982-1997). J Am Vet Med Assoc 2000;216:222-226

6. Abbas AK, Lichtman AH, Pober JS. Cellular and Molecular Immunology, 4th ed. Philadelphia, PA: WB Saunders; 2000:235-267.

7. Bookbinder PF, Butt MT, Harvey HJ. Determination of the number of mast cells in lymph node, bone marrow, and buffy coat cytologic specimens from dogs. J Am Vet Med Assoc 1992; 200:1648-1650.

8. McManus PM. Frequency and severity of mastocytemia in dogs with and without mast cell tumors: 120 cases (1995-1997). J Am Vet Med Assoc 1999;215:355-357.

9. Pollack MJ, Flanders JA, Johnson RC. Disseminated malignant mastocytoma in a dog. J Am Anim Hosp Assoc 1991;27:435440.

10. Davies AP, Hayden DW, Klausner JS, et al. Noncutaneous systemic mastocytosis and mast cell leukemia in a dog: Case report and literature review. J Am Anim Hosp Assoc 1981;17:361-368.

11. Lester SL, McGonigle LF, McDonald GK. Disseminated anaplastic mastocytoma with terminal mastocythemia in a dog. J Am Anim Hosp Assoc 1981;17:355-360.

12. Endicott MM, Charney SC, McKnight JA, et al. Clinicopathological findings and results of bone marrow aspiration in dogs 
with cutaneous mast cell tumours: 157 cases (1999-2002). Vet Comp Onc 2007;5:31-37.

13. Weiss DJ. A retrospective study of the incidence and the classification of bone marrow disorders in the dog at a veterinary teaching hospital (1996-2004). J Vet Intern Med 2006;20:955-961.

14. Mullins MN, Dernell WS, Withrow SJ, et al. Evaluation of prognostic factors associated with outcome in dogs with multiple cutaneous mast cell tumors treated with surgery with and without adjuvant treatment: 54 cases (1998-2004). J Am Vet Med Assoc 2006;228:91-95.

15. LaDue T, Price GS, Dodge R, et al. Radiation therapy for incompletely resected canine mast cell tumors. Vet Radiol Ultrasound 1998;39:57-62.

16. Spugnini EP, Vincenzi B, Baldi F, et al. Adjuvant electrochemotherapy for the treatment of incompletely resected canine mast cell tumors. Anticancer Res 2006;26:4585-4589.

17. Frimberger AE, Moore AS, Cincotta L, et al. Photodynamic therapy of naturally occurring tumors in animals using a novel benzophenothiazine photosensitizer. Clin Cancer Res 1998;4:22072218.

18. Thamm DH, Mauldin EA, Vail DM. Prednisone and vinblastine chemotherapy for canine mast cell tumor: 41 cases (19921997). J Vet Intern Med 1999;13:491-497.

19. Thamm DH, Turek MM, Vail DM. Outcome and prognostic factors following adjuvant prednisone/vinblastine chemotherapy for high-risk canine mast cell tumour: 61 cases. J Vet Med Sci 2006;68:581-587.

20. Hayes A, Adams V, Smith K, et al. Vinblastine and prednisolone chemotherapy for surgically excised grade III canine cutaneous mast cell tumours. Vet Comp Onc 2007;5:168-176.

21. Camps-Palau MA, Leibman NF, Elmslie R, et al. Treatment of canine mast cell tumours with vinblastine, cyclophosphamide and prednisone: 35 cases (1997-2004). Vet Comp Onc 2007;5:156167.

22. Rassnick KM, Moore AS, Williams LE, et al. Treatment of canine mast cell tumors with CCNU (lomustine). J Vet Intern Med 1999; 13:601-605.

23. Pryer NK, Lee LB, Zadovaskaya R, et al. Proof of target for SU11654: Inhibition of KIT phosphorylation in canine mast cell tumors. Clin Cancer Res 2003;9:5729-5734.

24. London CA, Hannah AL, Zadovoskaya R, et al. Phase I dose-escalating study of SU11654, a small molecule receptor tyrosine kinase inhibitor, in dogs with spontaneous malignancies. Clin Cancer Res 2003;9:2755-2768.

25. Webster JD, Yuzbasiyan-Gurkan V, Kaneene JB, et al. The role of c-KIT in tumorigenesis: Evaluation in canine cutaneous mast cell tumors. Neoplasia 2006;8:104-111.

26. London CA, Galli SJ, Yuuki T, et al. Spontaneous canine mast cell tumors express tandem duplications in the proto-oncogene c-kit. Exp Hematol 1999;27:689-697.

27. Downing S, Chien MB, Kass PH, et al. Prevalence and importance of internal tandem duplications in exons 11 and 12 of c-kit in mast cell tumors of dogs. Am J Vet Res 2002;63:1718-1723.
28. Zemke D, Yamini B, Yuzbasiyan-Gurkan V. Mutations in the juxtamembrane domain of c-KIT are associated with higher grade mast cell tumors in dogs. Vet Pathol 2002;39:529-535.

29. Webster JD, Kiupel M, Yuzbasiyan-Gurkan V. Evaluation of the kinase domain of c-KIT in canine cutaneous mast cell tumors. BMC Cancer 2006;6:85-92.

30. Webster JD, Yuzbasiyan-Gurkan V, Miller RA, et al. Cellular proliferation in canine cutaneous mast cell tumors: Associations with c-KIT and its role in prognostication. Vet Pathol 2007;44:298 308.

31. Gleixner KV, Rebuzzi L, Mayerhofer M, et al. Synergistic antiproliferative effects of KIT tyrosine kinase inhibitors on neoplastic canine mast cells. Exp Hematol 2007;35:1510-1521.

32. London C. Kinase inhibitors in cancer therapy. Vet Comp Onc 2004;2:177-193.

33. Kobie K, Kawabata M, Hioki K, et al. The tyrosine kinase inhibitor imatinib [STI571] induces regression of xenografted canine mast cell tumors in SCID mice. Res Vet Sci 2007;82:239-241.

34. Lachowicz JL, Post GS, Brodsky E. A phase I clinical trial evaluating imatinib mesylate (Gleevec) in tumor-bearing cats. J Vet Intern Med 2005;19:860-864.

35. Isotani $\mathrm{M}$, Tamura $\mathrm{K}$, Yagihara $\mathrm{H}$, et al. Identification of a c-kit exon 8 internal tandem duplication in a feline mast cell tumor case and its favorable response to the tyrosine kinase inhibitor imatinib mesylate. Vet Immunol Immunopathol 2006; 114:168-172.

36. Owen LN. TNM Classification of Tumors in Domestic Animals, 1st ed. Geneva, Switzerland: World Health Organization; 1980:7-15.

37. Cohen MH, Williams G, Johnson JR, et al. Approval summary for imatinib mesylate capsules in the treatment of chronic myelogenous leukaemia. Clin Cancer Res 2002;8:935-942.

38. Veterinary Cooperative Oncology Group. Veterinary cooperative oncology group - common terminology criteria for adverse events (VCOG-CTCAE) following chemotherapy or biological antineoplastic therapy in dogs and cats. Vet Comp Onc 2004;2:194 213.

39. Eckersall PD, Nash AS. Isoenzymes of canine plasma alkaline phosphatase: An investigation using isoelectric focusing and related to diagnosis. Res Vet Sci 1983;34:310-314.

40. Webster JD, Kiupel M, Kaneene JB, et al. The use of KIT and tryptase expression patterns as prognostic tools for canine cutaneous mast cell tumors. Vet Pathol 2004;41:371-377.

41. Stanclift RM, Gilson SD. Evaluation of neoadjuvant prednisone administration and surgical excision in treatment of cutaneous mast cell tumors in dogs. J Am Vet Med Assoc 2008; 232:53-62.

42. Gorre ME, Mohammed M, Ellwood K, et al. Clinical resistance to STI-571 cancer therapy caused by BCR-ABL gene mutation or amplification. Science 2001;293:876-880.

43. Pegram MD, Konecny GE, O'Callaghan C, et al. Rational combinations of trastuzumab with chemotherapeutic drugs used in the treatment of breast cancer. J Natl Cancer Inst 2004;96:739749. 